\section{Covid-19 nelle cure primarie pediatriche in Italia}

\section{Uno studio osservazionale, parte prima}

\author{
Giacomo Toffol \\ Pediatra, Pederobba (Treviso) \\ Roberto Buzzetti \\ Pediatra epidemiologo, Bergamo \\ Laura Reali \\ Pediatra, Roma
}

Introduzione: non esistono in letteratura molte pubblicazioni sulla storia clinica e la contagiosità dei casi pediatrici di Covid-19 non ospedalizzati. Per tale motivo abbiamo effettuato uno studio osservazionale nel setting della pediatria di famiglia italiana tra ottobre 2020 e marzo 2021.

Materiali e metodi: si tratta di uno studio osservazionale che ha arruolato: pazienti affetti da Covid-19; pazienti con sintomatologia sospetta ma con tampone negativo; contatti stretti di casi di Covid-19. II follow-up dei pazienti arruolati è stato di 28 giorni per i casi con diagnosi confermata, di 14 giorni per gli altri soggetti.

Risultati principali: sono stati arruolati 1947 pazienti di età inferiore a 14 anni: 465 con diagnosi di Covid-19; 549 soggetti con sintomatologia compatibile ma con tampone negativo; 933 contatti stretti di Covid-19. 130 soggetti con Covid-19 erano asintomatici al momento della diagnosi. Nei bambini di età inferiore a 3 anni il sintomo più frequente è stato la rinite (41\%), seguito da febbre (38\%) e tosse (31\%). In quelli di età compresa tra 3 e 14 anni il sintomo più frequente è stato la cefalea (30\% dei casi) seguito da rinite (23\%), astenia (18\%), febbre (16\%), tosse (11\%). Nessun sintomo considerato singolarmente è risultato in grado di far sospettare o escludere una diagnosi di malattia. L'analisi di regressione logistica multipla ha permesso di costruire uno score in grado di far sospettare la malattia nei bambini di età superiore a 2 anni, in base a: febbre, rash, rinite, cefalea, astenia.

Conclusioni: Lo studio ha confrontato soggetti con infezione da SARS-CoV-2 con soggetti non affetti ma con sintomatologia simile e ha confermato come sia impossibile in età pediatrica distinguere clinicamente il Covid-19 dalle altre patologie respiratorie. Lo score da noi calcolato, potenzialmente utile e valido per il periodo in cui si è svolto lo studio, non potrebbe essere applicato in altre situazioni epidemiologiche.

Introduction: Very few studies have been published on the clinical history and contagiousness of not hospitalized Covid-19 pediatric patients. Therefore, to get deeper in this topic, between October 2020 and March 2021, we carried out an observational study in the pediatric primary care setting in Italy.

Methods: The study enrolled patients with Covid-19 disease (cases); patients with suspicious symptoms but negative swab; close contacts of Covid-19 cases. The follow-up was 28 days for patients with Covid-19 disease, 14 days for the other subjects enrolled.

Results: 1947 patients aged 0-13 were enrolled: 465 with Covid-19 disease; 549 with suspicious symptoms but negative swab; 933 close contacts of Covid-19 cases. 130 subjects with Covid-19 were asymptomatic at the time of diagnosis. Among the others, in children aged 0-2 the most frequent symptom was rhinitis (41\%) followed by fever (38\%) and cough (31\%), In children aged 3-13 the most frequent symptom was headache (30\%) followed by rhinitis (23\%), asthenia (18\%), fever (16\%), cough (11\%). No symptoms alone were sufficient to induce or rule out the suspicion of the Covid-19 disease diagnosis. A multiple logistic regression analysis, conducted for the purpose, allowed to build a score capable of inducing disease suspicion in patients older than 2 years of age, based on: fever, rash, rhinitis, headache and asthenia.

Conclusions: This study compared subjects with SARSCoV-2 infection with unaffected subjects with similar symptoms and confirmed that it is impossible to clinically distinguish Covid-19 from other similar respiratory diseases in pediatric age. The obtained score, potentially useful and valid during the study period, could not be applied in other epidemiological scenarios.

\section{Introduzione}

La malattia Covid-I9 si è manifestata ufficialmente in Italia a febbraio 2020 e da allora si è diffusamente estesa causando decine di migliaia di vittime, prevalentemente nelle classi di età più avanzata [1]. A partire da gennaio 2020 sono comparse in letteratura le prime segnalazioni di casi pediatrici e neonatologici inizialmente provenienti dalla Cina e poi anche dagli altri Paesi in cui il contagio si è diffuso [2-4].

I casi pediatrici che inizialmente risultavano compresi tra l'I e il $5 \%$ dei casi totali, sono aumentati nel corso dei mesi, anche a seguito della parziale immunizzazione delle classi d'età più avanzate [5]. A metà settembre 2020, erano stati segnalati in Italia 9868 casi pediatrici di Covid-I9 (O-I8 anni) pari al $3,4 \%$ di tutti i casi [6]. A giugno 202I in Italia i casi pediatrici costituivano il $15,6 \%$ del totale $[7,8]$. Il decorso della malattia nell'età pediatrica è risultato significativamente meno grave rispetto alle età più avanzate. Dai dati riportati in letteratura si evidenzia un basso numero di ricoveri e una mortalità bassissima [9]. La storia clinica dei casi di Covid-I9 pediatrico che hanno necessitato di ricovero ospedaliero è stata ampiamente descritta da numerosi studi sia italiani sia internazionali $[10,11]$. Minori sono le informazioni relative al molto più grande numero di casi pediatrici meno gravi, che non necessitano di ospedalizzazione. Ancora non esaustive sono inoltre le informazioni sulla contagiosità del virus SARS-CoV-2 in questa fascia d'età e sul ruolo che giocano i bambini e gli adolescenti nella trasmissione del virus.

Per tali motivi abbiamo programmato, al termine della prima ondata di Covid-I9 in Italia, uno studio osservazionale con l'intento di valutare l'incidenza di Covid-I9 e dei fattori a essa associati in una popolazione pediatrica nel setting delle cure primarie. Lo studio è stato effettuato attraverso l'acquisizione di dati sul decorso clinico di: pazienti pediatrici affetti da Covid-I9, con diagnosi confermata mediante tampone molecolare; casi sospetti con tampone negativo; contatti stretti familiari o extrafamiliari di casi di Covid-I9.

\section{Materiali e metodi}

Si è trattato di uno studio osservazionale multicentrico. I dati necessari allo studio sono stati raccolti negli ambulatori dei pediatri di famiglia, che in Italia si occupano della cura di tutta la popolazione in età pediatrica Lo studio ha previsto l'arruolamento di tre categorie di pazienti di età O-I3 anni (fino a 
I4 non ancora compiuti): pazienti affetti da Covid-I9, con diagnosi confermata mediate tampone molecolare; pazienti con sintomatologia sospetta per infezione da SARS-CoV-2 ma con tampone negativo; contatti stretti familiari o extrafamiliari di casi di Covid-I9. I pazienti con queste caratteristiche entrati in contatto con i pediatri sperimentatori sono stati arruolati nello studio dopo aver firmato un apposito consenso informato. La partecipazione allo studio non prevedeva alcuna analisi supplementare ma solo la raccolta degli abituali dati anamnestici e clinici: età, sesso, peso, altezza, condizioni cliniche generali, patologie croniche, tosse, febbre, dispnea, vomito, diarrea, lesioni dermatologiche, sintomi neurologici, muscolari, cardiocircolatori. Son state inoltre raccolte informazioni clinico-anamnestiche sui familiari. Tutti i pazienti sono stati seguiti secondo i protocolli previsti per il trattamento di questa malattia. Il follow-up dei pazienti arruolati è stato effettuato mediante interviste telefoniche e mediante la compilazione di appositi questionari online. La durata del follow-up è stata di 28 giorni per i casi di Covid-I9, di I4 giorni per gli altri soggetti. L'arruolamento dei pazienti è stato condotto tra il I8 ottobre 2020 e il 30 marzo 202I. Lo studio è stato approvato dal Comitato Etico dell'ULSS 2 Veneto (Treviso) in settembre 2020. L'endpoint primario dello studio era la stima dell'incidenza della malattia nella popolazione seguita dai pediatri che hanno aderito allo studio. Obiettivi secondari erano: descrivere la sintomatologia di questa malattia nei soggetti positivi e valutare l'applicabilità di una scheda di sintomi clinici per orientare verso il sospetto di diagnosi di Covid-I9. Sono stati valutati i sintomi più frequenti ed è stata calcolata la loro sensibilità e specificità se presi singolarmente, confrontandone la frequenza nei positivi e nei negativi al tampone. Con l'intento di chiarire quali elementi sintomatologici siano più associati con la diagnosi di Covid-I9, dopo aver diviso gli arruolati in due classi di età (0-2 = meno di 3 anni; $3-\mathrm{I} 3=$ più di 3 anni e meno di I4), per entrambe è stata condotta un'analisi di regressione logistica multipla, che ha considerato come outcome la diagnosi di Covid-I9 e come variabili predittive tutti i segni/sintomi rilevati. Dopo aver analizzato il modello completo sono state via via eliminate le variabili non significativamente associate con la diagnosi. Il modello logistico finale ha permesso di costruire uno score per il calcolo della probabilità di diagnosi (valore predittivo) per ognuna delle diverse combinazioni di presenza o assenza dei sintomi.

\section{Risultati}

Hanno partecipato allo studio 34 pediatri di famiglia aderenti all'Associazione Culturale Pediatri, adeguatamente formati nell'estate 2020 (https://acp.it/assets/media/Elenco_alfae betico_partecipanti_studio_CovidI9.pdf). La popolazione seguita dai pediatri partecipanti all'inizio dello studio era di 33.I79 bambini, di cui I3.720 con età inferiore a 6 anni. La distribuzione regionale dei pediatri partecipanti e quindi della popolazione in studio non è rappresentativa della realtà italiana, ma si è concentrata in modo particolare in alcune Regioni: Veneto (Io pediatri, I0.283 bambini), Lombardia $(8,7390)$, Piemonte $(6,5967)$, Sicilia $(4,3675)$. Le altre Regioni coinvolte sono state Campania (2 pediatri, I827 bambini), Friuli, Puglia, Emilia-Romagna e Lazio (I pediatra per Regione con rispettivamente IIO8, IO50, 990 e 889 bambini).

Il numero totale di bambini arruolati nello studio è stato di I947, così suddivisi: 465 soggetti con diagnosi di Covid-I9 confermata da tampone molecolare; 549 soggetti con sintomatologia compatibile con la malattia ma con tampone negativo; 933 contatti stretti di Covid-I9, in ambito familiare o scolastico. L'età media dei soggetti arruolati era di 6,4 anni [Figura 1]. I 465 soggetti con diagnosi di Covid-I9 confermata sono distribuiti in tutte le classi d'età, ma il rapporto tra i soggetti positivi e negativi tende ad aumentare al crescere dell'età.

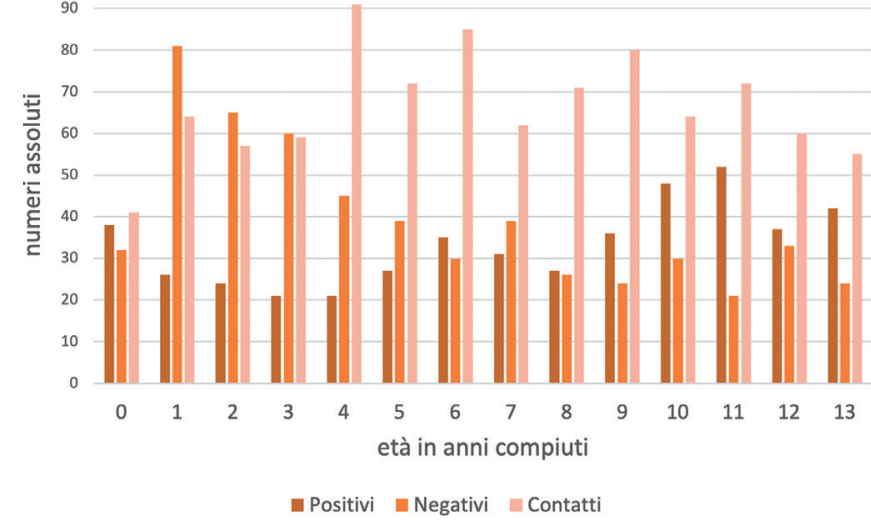

Figura 1. Età dei soggetti arruolati (positivi e negativi al tampone molecolare per SARS-CoV-2 e contatti).

L'incidenza complessiva dei casi di malattia nella popolazione seguita è stata di 3,8 casi/Iooo bambini/mese (3,I per i bambini $0-5$ anni e 4,3 per i bambini 6-I3 anni) con differenze significative tra le Regioni [Tabella 1]. Emilia-Romagna e Veneto sono state le Regioni con incidenza più alta (rispettivamente II,2 e 5,I), mentre tutte le altre Regioni hanno avuto un'incidenza compresa tra I e 3,2. Queste differenze sono verosimilmente da attribuirsi alla diversa gestione della malattia nelle varie Regioni, con diversa possibilità di confermare la diagnosi nei casi sospetti.

Tabella 1. Incidenza dei casi di Covid-19 nei 6 mesi dello studio (casi/1000 bambini/mese)

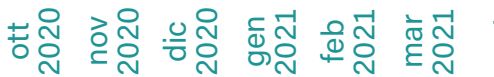

\begin{tabular}{llllllll}
\hline incidenza < 6 anni & 2,7 & 2,3 & 4,2 & 3,5 & 2,2 & 3,3 & 3,1 \\
incidenza > 6 anni & 7,0 & 5,4 & 4,6 & 3,3 & 3,1 & 3,3 & 4,3 \\
incidenza totale & 5,3 & 4,1 & 4,4 & 3,4 & 2,7 & 3,3 & 3,8
\end{tabular}

Le malattie croniche sono scarsamente rappresentate nel campione di pazienti arruolato e non sembrano influenzare la possibilità di avere una diagnosi positiva. Complessivamente sono affetti da malattia cronica il 7,6\% degli arruolati e il 9,2\% dei positivi. Tra le principali segnaliamo l'asma ( $2 \%$ dei pazienti), le malattie neurologiche e i disturbi del neuro-sviluppo (I,2\%), le malformazioni/malattie congenite $(0,9 \%)$, le cardiopatie (0,7\%). Numerosi sono invece, quasi I su 5 , i bambini esposti a fumo passivo nelle proprie abitazioni. Si tratta del I8,4\% del totale, con differenze importanti tra le varie Regioni italiane. Si passa infatti dal 34,5\% del Lazio e dal 32\% della Campania al I4,7\% del Veneto e al I3,3\% dell'Emilia-Romagna. Non tutti i bambini affetti da Covid-I9 erano sintomatici al momento della diagnosi, in quanto secondo il protocollo dello studio sono stati arruolati tutti i soggetti con tampone molecolare positivo, indipendentemente dal motivo di esecuzione del tampone. Tra i soggetti arruolati con tampone positivo I30 erano completamente asintomatici al momento della diagnosi. Di altri 62 soggetti non sono stati raccolti i sintomi. I rimanenti soggetti (373 bambini) presentavano diversi sintomi, variamente combinati. L'analisi dei segni e dei sintomi è stata effettuata suddividendo i soggetti positivi in due classi d'età: 0-2 (prima dei tre anni alcuni sintomi erano impossibili da riferire) e 3-I3. Negli 88 bambini di età $0-2$ il sintomo più frequente è stato la rinite $(4 \mathrm{I} \%)$ seguito dalla febbre (temperatura $\geq \operatorname{di~} 38^{\circ} \mathrm{C}, 38 \%$ ) e dalla tosse (3I\%). Altri sintomi presenti erano: diarrea (I5\%), vomito (6\%), congiuntivite $(6 \%)$, rash $(5 \%)$, tachipnea/dispnea (3\%), acrocianosi (I\%). Nei più grandi 


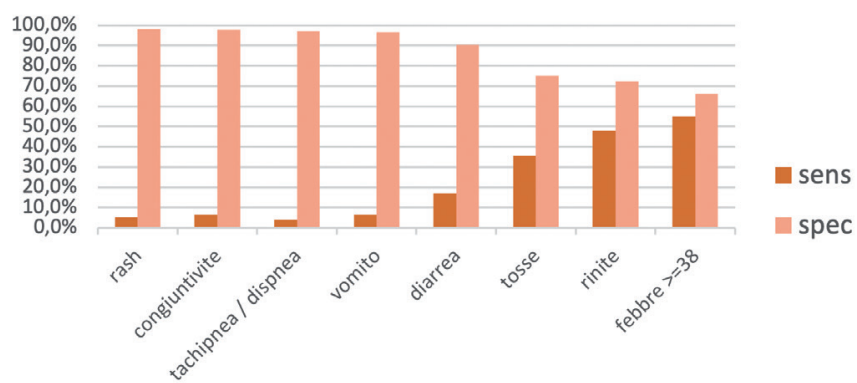

Figura 2. Sensibilità e specificità dei più frequenti segni clinici e sintomi nei bambini di età inferiore a 3 anni.

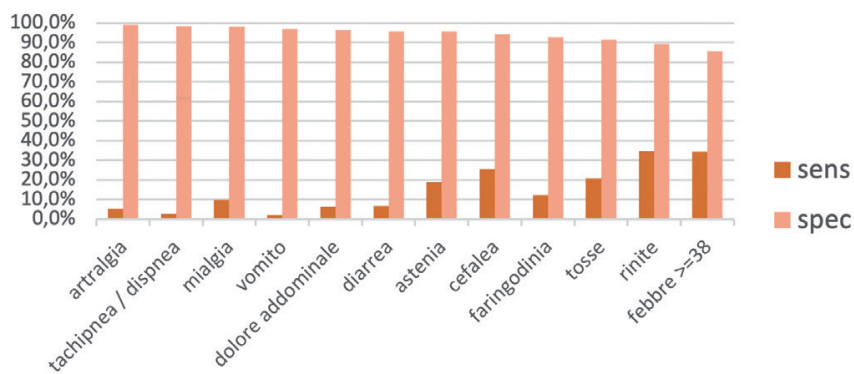

Figura 3. Sensibilità e specificità dei più frequenti segni clinici e sintomi nei bambini di età 3-13 anni.

(377 bambini di età 3-I3 anni) il sintomo più frequente è stato la cefalea (30\%) seguito da rinite (23\%), astenia (I8\%), febbre (I6\%), tosse (II\%), faringodinia (8\%), mialgia (6\%), diarrea $(6 \%)$, dolore addominale $(5 \%)$ e artralgia $(4 \%)$. Ageusia, tachipnea/dispnea, dolore oculare, anosmia, rash, congiuntivite erano presenti ognuno nel $2 \%$ dei casi, mentre vomito, dolore toracico e acrocianosi erano presenti nell'ı\% dei casi. Solo per 7 bambini, pari all'ı, $\%$ degli arruolati, è stato necessario il ricovero ospedaliero.

La frequenza dei sintomi di esordio è stata confrontata nei due gruppi di soggetti arruolati, sintomatici con diagnosi confermata e sintomatici con esclusione diagnostica di Covid-I9. Nessun sintomo considerato singolarmente è risultato in grado di far sospettare una diagnosi di malattia, con l'esclusione di ageusia, acrocianosi e lesioni ulcerative delle estremità, presenti però in un numero estremamente basso di pazienti. Nessun sintomo inoltre è stato utile per permettere un'esclusione clinica della malattia. Nelle Figure 2,3 sono riportati i valori di sensibilità e specificità dei sintomi più comuni ottenuti mettendo a confronto i soggetti positivi al Covid-I9 con tutti gli altri arruolati.

Tabella 2. Score per stimare la probabilità di diagnosi di Covid-19 a partire dai sintomi di esordio: istruzioni per l'uso

In presenza di queste condizioni Aggiungere il punteggio

Per ogni anno di età

Temperatura $\geq 38^{\circ} \mathrm{C}$

Rinite

8

Rash 14

Astenia

Cefalea 8

Al totale vanno poi sottratti 2 punti

Secondo l'analisi multipla, nei bambini al di sotto dei 3 anni l'unica variabile significativamente associata con la diagnosi di Covid-I9 sarebbe la rinite (OR 2,35 IC 95\% da I,09 a 5,08).
Più interessante il risultato nella classe di età superiore a 3 anni. Da questa analisi è stato possibile ricavare uno score, riportato in Tabella 2, così strutturato: si assegna I punto per ogni anno di età; 4 punti per la presenza di febbre $\left(\geq 38^{\circ} \mathrm{C}\right) ; 4$ punti per l'astenia; 8 punti per presenza di rinite; 8 punti per la cefalea; I4 punti per il rash; infine, si sottraggono 2 punti. Questo score può assumere valori tra un minimo di I e un massimo di 49. La Figura 4 presenta una curva che permette di passare

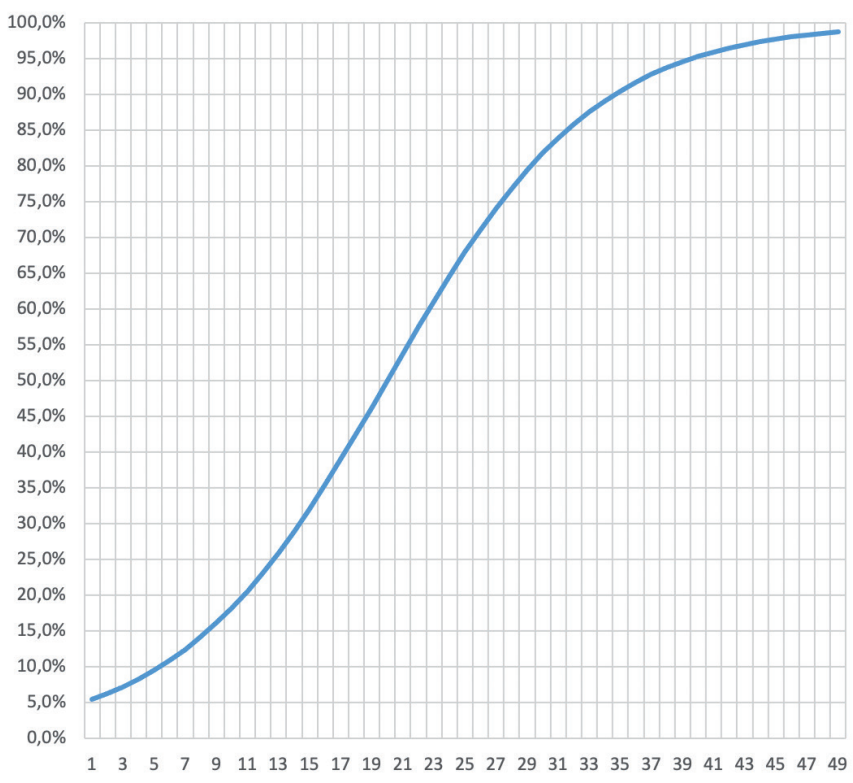

Figura 4. Stima della probabilità di Covid-19 in base allo score.

dal punteggio ottenuto (ascissa) alla probabilità di Covid-I9 (ordinata). Volendo esemplificare, un bambino di 5 anni con febbre, rinite, astenia e cefalea avrebbe un punteggio $=27$, e una probabilità di Covid-I9 pari al 75\% circa. Larea sotto la curva ROC è $=0,729$.

\section{Discussione}

Il periodo di arruolamento dello studio (ottobre 2020-marzo 202I) si è sovrapposto esattamente alla seconda e terza ondata della malattia in Italia, permettendo un'osservazione del fenomeno in tempo reale. Ciò ha comportato però difficoltà impreviste da parte dei ricercatori, che si sono trovati a operare in un momento di intenso stress lavorativo, e con indicazioni operative variabili e spesso diverse a seconda delle Province e Regioni italiane. Non possiamo pertanto escludere una sottostima del campione dei positivi, per possibile non completa esaustività della raccolta dati. Le incidenze rilevate sono state massime in Emilia-Romagna e Veneto rispetto alle altre Regioni e questo è sicuramente dipeso, oltre che dall'entità reale del fenomeno, anche dalle modalità diagnostiche definite dai servizi di igiene pubblica di queste Regioni, che hanno permesso ai pediatri di richiedere e ottenere in tempi rapidi un maggior numero di tamponi per il SARS-CoV-2 identificando quindi un maggior numero di pazienti. Nonostante queste difficoltà e diversità regionali il numero di pazienti positivi arruolati dai medici ricercatori è stato significativamente elevato, e ha permesso di confermare come la malattia interessa anche l'età pediatrica con incidenza maggiore al crescere dell'età.

Il nostro studio ha confermato come nei bambini la malattia sia raramente grave e il ricovero in ospedale sia infrequentemente necessario. Come già evidenziato da altri studi sospettare clinicamente questa malattia in età pediatrica è molto difficile, in quanto i sintomi si sovrappongono a quelli delle più comuni malattie respiratori dei bambini [12]. La rinite, la febbre e la tosse si sono dimostrati i sintomi più frequenti 
nei bambini piccoli, mentre nei bambini più grandi e nei ragazzi a questi si sono associati cefalea e astenia, confermando quanto già noto $[13,14]$. Gli unici sintomi in grado di orientare una diagnosi si manifestano solo in una esigua percentuale di bambini e sono quindi di scarso aiuto pratico per individuare tale patologia. Risulta di contro evidente l'utilità dello score da noi calcolato; va però osservato che la sua validità al di là del periodo in cui si è svolto lo studio è probabilmente scarsa, date le mutate condizioni epidemiologiche.

\section{Conclusioni}

Questo articolo presenta i dati iniziali di uno studio osservazionale svolto negli ambulatori dei pediatri di famiglia e descrive le caratteristiche cliniche dell'esordio della malattia Covid-I9, confrontando soggetti con diagnosi confermata di infezione da SARS-CoV-2 con soggetti negativi ma con sintomatologia simile. A nostra conoscenza rappresenta la prima pubblicazione del genere in Italia, e ha permesso di confermare come sia quasi impossibile in età pediatrica distinguere clinicamente questa patologia dalle altre patologie virali respiratorie dei bambini. I dati sul follow-up della infezione da SARS-CoV-2 nella popolazione in esame, ancora in fase di analisi, saranno oggetto di una successiva pubblicazione. Ringraziamo tutti i pediatri che hanno reso possibile la realizzazione di questo studio superando tutte le difficoltà emerse durante la fase epidemica.

\section{Bibliografia}

1. Livingston E, Bucher K. Coronavirus Disease 2019 (Covid-I9) in Italy. JAMA. 2020 Apr I4;323(I4):I335.

2. Dong $\mathrm{Y}, \mathrm{Mo} \mathrm{X}, \mathrm{Hu} \mathrm{Y}$, et al. Epidemiology of Covid-I9 Among Children in China. Pediatrics. 2020 Jun;I45(6):e2O200702.

3. CDC Covid-I9 Response Team. Coronavirus Disease 2019 in Chil dren - United States, February I2-April 2, 2020. MMWR Morb Mortal Wkly Rep. 2020 Apr IO;69(I4):422-426.
4. Castagnoli R, Votto M, Licari A, et al. Severe Acute Respiratory Syndrome Coronavirus 2 (SARS-CoV-2) Infection in Children and Adolescents: A Systematic Review. JAMA Pediatr. 2020 Sep I;I74(9):882-889.

5. Ludvigsson JF. Systematic review of Covid-I9 in children shows milder cases and a better prognosis than adults. Acta Paediatr. 2020 Jun;I09(6):Io88-1095.

6. Bellino S, Rota MC, Riccardo F, et al. Pediatric Covid-I9 Cases Prelockdown and Postlockdown in Italy. Pediatrics. 202I Feb;I47(2):e2020035238.

7. ISS. Bollettino sorveglianza integrata Covid-I9, Aggiornamento nazionale II agosto 202I. https://www.epicentro.iss.it/coronavirus/bollettif no/Bollettino-sorveglianza-integrata-Covid-I9_II-agosto-202I.pdf

8. Tanne JH. Covid-I9: Cases in children rise sharply in US as doctors call for vaccine approval. BMJ. 202I Aug I6;374:n2030.

9. Bhopal SS, Bagaria J, Olabi B, Bhopal R. Children and young people remain at low risk of Covid-I9 mortality. Lancet Child Adolesc Health. 202I May;5(5):eI2-eI3.

10. Cella A, Marchetti F, Iughetti L, et al. Italian Covid-I9 epidemic: ef fects on paediatric emergency attendance-a survey in the Emilia Romagna region. BMJ Paediatr Open. 2020 Jul 20;4(I):e000742.

11. Parri N, Lenge M, Buonsenso D. Children with Covid-I9 in Pediatric Emergency Departments in Italy. N Engl J Med. 2020 Jul 9;383(2):I87-I9O.

12. Poline J, Gaschignard J, Leblanc C, et al. Systematic Severe Acute Respiratory Syndrome Coronavirus 2 Screening at Hospital Admission in Children: A French Prospective Multicenter Study. Clin Infect Dis. 202I Jun I5;72(I2):2215-22I7.

13. Nikolopoulou GB, Maltezou HC. Covid-I9 in children: where do we stand? Arch Med Res. 202I Jul 6;SoI88-4409(2I)ooI48-X.

14. Molteni E, Sudre CH, Canas LS, et al. Illness duration and symptom profile in symptomatic UK school-aged children tested for SARSCoV-2. Lancet Child Adolesc Health. 202I Oct;5(IO):708-7I8.

\section{Attendere prima di prendere l'antibiotico è meglio}

\section{blister}

Le infezioni delle vie respiratorie sono pane quotidiano per il pediatra delle cure primarie e gran parte delle richieste di visita per patologia sono rivolte a questo problema. II medico, dopo aver visitato il bambino, può decidere di prescrivere l'antibiotico indicando di somministrarlo subito, oppure di ritardare l'inizio della terapia se i sintomi persistono dopo uno, due o più giorni di attesa, secondo la situazione clinica. Che cosa funziona meglio? Sono stati fatti molti studi su questo argomento e una revisione con metanalisi è recentemente stata pubblicata sul BMJ [1]. Gli autori di questa revisione sistematica hanno selezionato i trial per conoscere la severità dei sintomi (esito primario) a 2 e a 4 giorni dopo la vista dal medico e (esito secondario) la durata complessiva dei sintomi, le complicanze, gli accessi in ospedale o ulteriori visite dal medico, l'evento morte e la soddisfazione dei pazienti. È meglio dunque la prescrizione immediata dell'antibiotico oppure è bene ritardare? La revisione ha raccolto 9 RCT e 4 studi osservazionali (55.682 pazienti tra bambini e adulti) e non ha rilevato differenze nell'esito primario sia per prescrizione immediata vs ritardata (differenza media aggiustata $-0,003$, IC $95 \%-0,12,0,11)$ che per prescrizione ritardata vs nessuna prescrizione $(0,02, I C 95 \%-0,11,0,15)$. La durata dei sintomi è lievemente più lunga nel caso in cui si attenda a dare l'antibiotico vs prescrizione immediata, anche se a livello clinico la differenza è poco importante (11,4 vs 10,9 giorni), mentre non ci sono differenze tra prescrizione ritardata vs nessuna prescrizione. E per gli altri esiti secondari? Attendere prima di dare l'antibiotico riduce le complicazioni, gli accessi in ospedale, le successive consultazioni dal curante e, infine, aumenta la soddisfazione del paziente. Una metanalisi effettuata su bambini di età 0-4 anni ha rilevato una maggiore persistenza dei sintomi tra 2 e 4 giorni in caso di prescrizione ritardata vs immediata con una differenza significativa dal punto di vista statistico $(0,10, I C 95 \% 0,03,0,18)$; ma anche in questo caso, considerando che la differenza di 0,10 è su una scala da 0 a 6 in cui ogni punto è equivalente a un partecipante su 10 con sintomi, si può dire che clinicamente il dato è di scarso significato.

Questa revisione conferma gli esiti di un recente RCT [2] svolto su pazienti pediatrici (presentato nelle "Pagine elettroniche" di Quaderni acp (https://acp.it/assets/media/Quaderni_acp_2021_284_PE_n1.pdf). Quindi fare meno, ossia ritardare la pret scrizione di antibiotico nelle infezioni delle vie respiratorie, è meglio: si risparmia sul consumo di antibiotici e in più i bambini hanno meno complicanze e meno visite.

1. Stuart B, Hounkpatin $H$, Becque $T$, et al. Delayed antibiotic prescribing for respiratory tract infections: individual patient data meta-analysis. BMJ. 202I Apr 28;373:n808.

2. Mas-Dalmau G, Villanueva López C, Gorrotxategi Gorrotxategi P, et al. Delayed Antibiotic Prescription for Children With Respiratory Infections: A Randomized Trial. Pediatrics. 202I Mar;I47(3):e2O2OI323. 